EPiC Series in Engineering
Volume 2, 2018, Pages 67-81
SUMO 2018- Simulating Autonomous
and Intermodal Transport Systems

\title{
Chula-SSS: Developmental Framework for Signal Actuated Logics on SUMO Platform in Over-saturated Sathorn Road Network Scenario
}

\author{
Chaodit Aswakul ${ }^{1}$, Sorawee Watarakitpaisarn ${ }^{1}$, Patrachart Komolkiti ${ }^{2}$, Chonti \\ Krisanachantara ${ }^{3}$, and Kittiphan Techakittiroj ${ }^{3}$
}

1 Wireless Network and Future Internet Research Unit, Department of Electrical Engineering, Faculty of Engineering, Chulalongkorn University, Bangkok 10330, Thailand.

chaodit.a@chula.ac.th, james628_sw@hotmail.com

2 Learning Innovation Center, Chulalongkorn University, Bangkok 10330, Thailand. patrachart.k@chula.ac.th

3 Sathorn Model Project, Faculty of Engineering, Chulalongkorn University, Bangkok 10330, Thailand.

u5517443@au.edu, ktech@talent.in.th

\begin{abstract}
In this paper, Chula-Sathorn SUMO Simulator (Chula-SSS) has been proposed as an educational tool for traffic police and traffic engineers. The tool supports our framework to develop actuated traffic signal control logics in order to resolve urban traffic congestion. The framework design aims to incorporate the tacit traffic control expertise of human operators by trying to extract and extend the human-level intelligence in actuating logically traffic signal controls. In this regard, a new software package has been developed for the microscopic-mobility computer simulation capability of the SUMO (Simulation of Urban MObility) platform. Using the SUMO TraCI, our package implements the graphical user interface (GUI) of actual traffic light signal control panel, recently introduced in Bangkok (Thailand) for traffic police deployment in the Chulalongkorn University's Sathorn Model project under the umbrella of Sustainable Mobility Project 2.0 of the World Business Council for Sustainable Development (WBCSD). The traffic light signal control panel GUI modules can communicate via TraCI in real-time to SUMO in order both to retrieve the raw traffic sensor data emulated within SUMO and to send the desired traffic light signal phase manually entered via GUI by the module users. Each of the users could play a role of traffic police in charge of actuating the traffic light signal at each of the controllable intersections. To demonstrate this framework, Chula-SSS has been implemented with the calibrated SUMO dataset of Sathorn Road network area. This area is one of the most critical areas in Bangkok due to the immense traffic volume with daily recurring traffic bottlenecks and network deadlocks. The simulation comprises of 2375 intersection nodes, 4517 edges, 10 main signalised intersections. The provided datasets with Chula-SSS cover both the morning and evening rush-hour periods each with over 55,000 simulated vehicles based on the comprehensive traffic data collection and SUMO mobility model calibration. It is hoped that the herein developed framework and software package can be not only
\end{abstract}

E. Wießner, L. Lücken, R. Hilbrich, Y.-P. Flötteröd, J. Erdmann, L. Bieker-Walz and M. Behrisch (eds.), SUMO2018 (EPiC Series in Engineering, vol. 2), pp. 67-81 

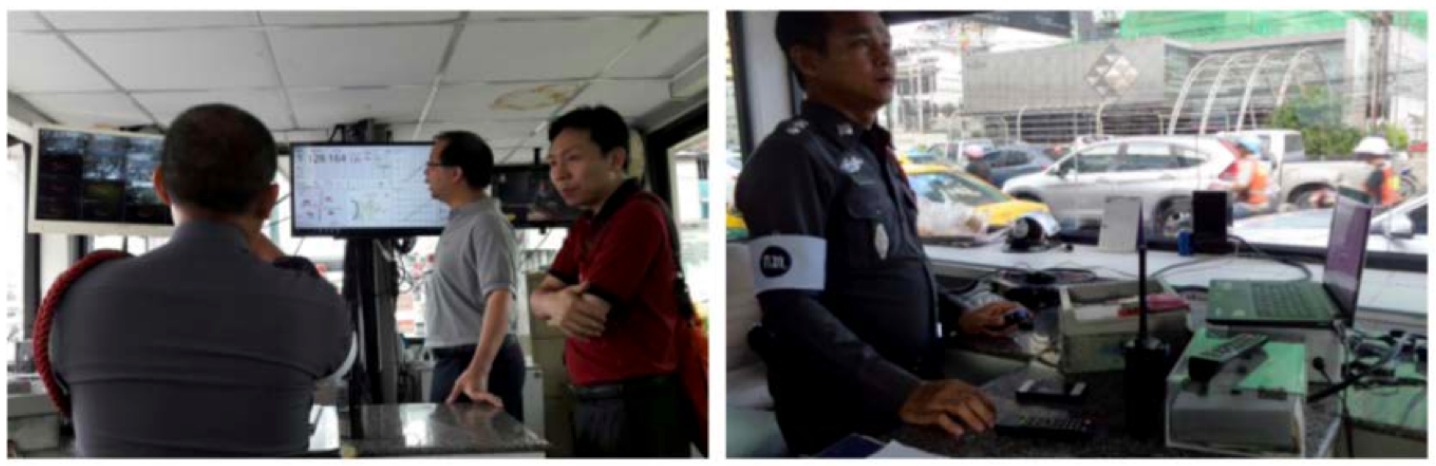

Figure 1: Local traffic control room at Sathorn Intersection.

useful for our Thailand case, but also readily extensible to those developing and leastdeveloped countries where traffic signal controls rely on human operations, not yet fully automated by an area traffic controller. In those cases, the framework proposed herein is expectedly an enabling technology for the human operators to practice, learn, and evolve their traffic signal control strategies systematically.

\section{Introduction}

In cities of developing countries around the world, standard practices of traffic police for controlling traffic signals are limited in many aspects. Due to high installation and operational cost, scarcely available real-time traffic sensors are insufficient and that renders automatic controls impractical. As a result, trial-and-error manual operations are commonly practiced within the actual road networks. Arguably, those operations are advantageous in comparison with other networked control-theoretic approaches. Particularly, all the trial-and-error logics can be applied and evaluated directly in the real system. In doing so, one needs not concerned of unjustified theoretical assumptions. However, there are several drawbacks concerning those standard trial-and-error practices.

Firstly, the traffic signal operation and demand conditions are unrepeatable. The responsible traffic police cannot recall conveniently their past practices, should they want to find out how much they can gain by modifying the previous traffic signal operation strategy. Secondly, traffic police in many cities would rely on CCTVs to observe traffic flow movements so as to adjust their traffic light signals. The traffic police have to approximate the queue lengths by watching many CCTVs simultaneously shown on their screen (as an example depicted in Figure 1). This observation process can be done at best in developing countries merely subjectively. In addition, due to the limited, often insufficient number of CCTVs and the available view angles, traffic police resort to guess on the corresponding vehicle queues. All these occasionally lead to inappropriate decisions on giving the right of way to conflicting vehicle directions.

Every decision traffic police make has direct impacts on drivers. If the trial-and-error logics are not optimal in actuating traffic light signals, then detrimental effects will be upon the real road networks and commuters. On the other hand, there are evidences suggesting that there is a clear tacit knowledge accumulated by traffic police expertise. That knowledge should be extracted systematically and made a practice standard. In this regard, there is a challenging need to develop a systematic framework whereby traffic signal operations can be tried out, 


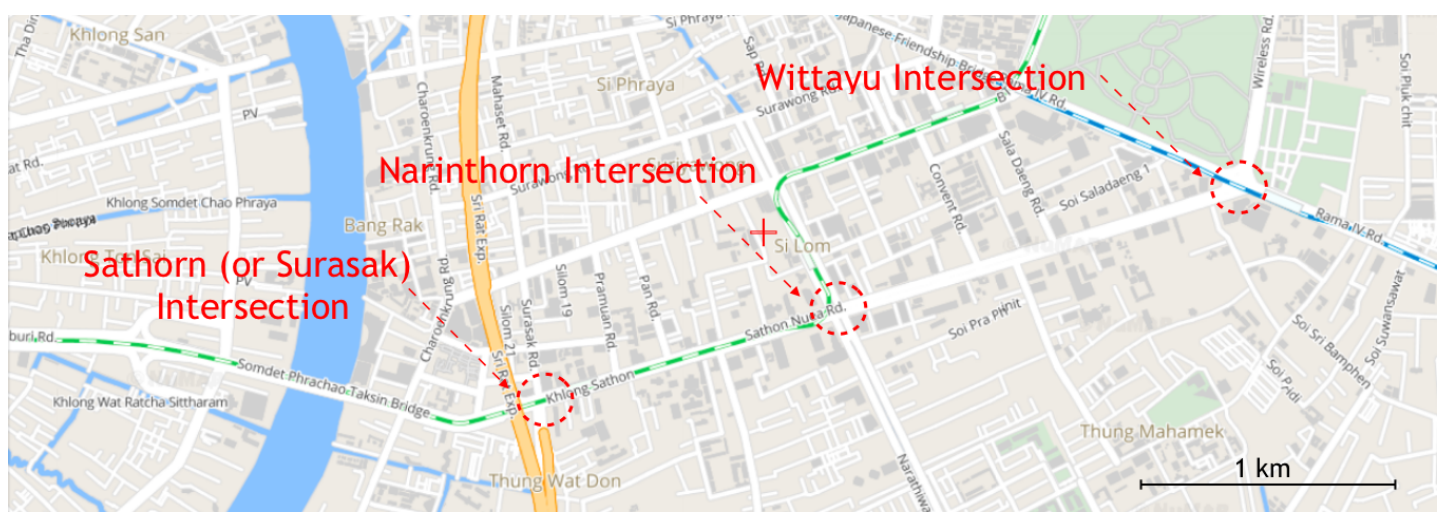

Figure 2: Map of Sathorn Road network (with granted usage permission from http://map.longdo.com/).

repeated, learnt and improved without causing problems to the real road network users.

As an attempt to address the challenge aforementioned, the Traffic Flow Management and Bottleneck Mitigation Workgroup has been established in the Chulalongkorn University's Sathorn Model project. This project has been carried out under the globally initiative umbrella of Sustainable Mobility Project 2.0 of the World Business Council for Sustainable Development (WBCSD) [16]. In our Sathorn Model project, the target test site is the network of Sathorn Road in Bangkok, Thailand. Based on the 2016 traffic index report [10], Bangkok retains the first ranking of the most congested city in Asia and the second ranking globally. The daily extra travel time as much as 64 minutes has been reported in comparison with the travel time that would be required during a free-flow condition [10]. Dealing with the Bangkok traffic congestion problem is highly challenging. During weekdays, Bangkok road networks are usually found over-saturated, and bottlenecks as well as deadlocks become common events.

In fact, amongst all the districts within Bangkok, the Sathorn Road network has been reported as the most congested one with the lowest travel speed [11]. This is because of all the high-rise buildings along side Sathorn Road, with only half of the road segment serviceable by the convenient public transport i.e. the sky train and subway systems. Further, Sathorn Road serves as the arterial gateway linking between the residential area on the western side of Chao Phraya River and the business area on the river-eastern side, as shown in Figure 2. As a result, up to 150,000 vehicles drive every weekday on Sathorn Road [16]. Based on our sensors installed at the Sathorn Intersection, the morning congestion period during weekdays start from $6 \mathrm{am}$ to $9 \mathrm{am}$ and the evening congestion period starts from $3 \mathrm{pm}$ till $7 \mathrm{pm}$ with more than 55,000 traveling vehicles during the morning as well as evening rush hours.

The framework in this paper has been developed by using Simulation of Urban MObility (SUMO) [4] as the simulation and visualisation platform. SUMO is used in our framework because it is a well-known microscopic simulator software. Microscopic behaviour of vehicles can be beneficial to simulate flows of stopping vehicles in the real situation on Sathorn Road. This is advantageous when comparing with the usage of macroscopic simulators, where those detailed movement and bottleneck forming effects cannot as well be captured since moving vehicles would be treated instead as fluid flows. Furthermore, SUMO is an open-source software. This characteristic benefits developers in terms of its extensibility and allows us the choice to distribute further our developed source codes.

By using SUMO traffic simulator capability, traffic police can repeatedly work on exactly the 
same traffic scenarios by setting a constant initial seed value of random number generator. The bird-eye view of SUMO-GUI displays an overall view of traffic demands and congestion patterns. This richness of visualisation provides traffic police with both local and global information to improve their traffic signal logics and strategies. In this paper, the so-called Chula-Sathorn SUMO Simulator (Chula-SSS) has been proposed as an educational tool for traffic police and traffic engineers. The tool supports the intended framework to facilitate the development of actuated traffic signal control logics in order to resolve urban traffic congestion. The framework design aims to incorporate the tacit traffic control expertise of human operators of traffic light signals by trying to extract and extend the human-level intelligence in actuating logically traffic signal controls. In addition, for the validity of the Chula-SSS simulated scenarios, a brute-force calibration process has been carried out by varying microscopic simulation parameters of SUMO in order to make the behaviour of vehicle flows in the simulation as close to that in the real road network as possible. Both Chula-SSS source codes and the underlying Sathorn Road network datasets during morning/evening rush hours are made publicly downloadable with this publication.

\section{Proposed Chula-Sathorn SUMO Simulator (Chula-SSS)}

Chula-SSS is proposed as an educational tool mainly for traffic police and traffic engineers to find optimal measures in order to resolve traffic congestion in the Sathorn area. The overall layout of equipment settings in Chula-SSS is depicted in Figure 3. A computer notebook runs the new software package, Chula-SSS server. The server program is responsible for starting the standard SUMO-GUI software with our Sathorn Road network datasets as well as adjusting the SUMO clock timing to compensate for the computer notebook hardware CPU speed. This clock tuning should allow the module users to see the simulated vehicle movement as close as possible to real time. And if desired, the Chula-SSS server can also be configured to run at a higher speed than the real time with the clock-scaling factor of $1,2,4,8,16,32,64$ and 128 times.

The Chula-SSS server displays its output at a big flat-panel television screen. Thus, all module users can share the visibility of simulated road network condition. The users can then consult with each other conveniently in developing new signal actuating logics together. Figure 4 depicts a snapshot of Chula-SSS server display. Here, the top window shows the overall Sathorn Road network. The bottom windows show three main signalised intersections on the road, namely, Sathorn-Narinthorn-Wittayu Intersections at the bottom left-middle-right windows, respectively. Users can use the overall window at the top part to see queue lengths. Users can use the individual signalised intersection windows at the bottom part to see the current phases and localised congestion situation so as to decide on switching traffic light signal phases.

To change the traffic signal phases of main signalised intersections, the users can use the Chula-SSS client programs installed within the Windows ${ }^{\circledR}$ tablet computers. With TraCI [15] allowing the accessibility of SUMO simulator as a networked server, the developed Chula-SSS clients are all connected to the Chula-SSS server within a single wireless local area network. The Chula-SSS server and client programs are connected by UDP/IP. So, all Chula-SSS clients are configured to connect directly with the IP address of the Chula-SSS server.

Users especially traffic police may not be trained to use complex tools. For that reason, Chula-SSS design objective is to have user-friendly interfaces that have already been familiar to those target users. Therefore, at the Chula-SSS client user interface of a selected intersection, the displayed traffic signal operation panel (shown in Figure 5) has been designed to mimic the 


\section{(2) Full-HD Big TV Screen}

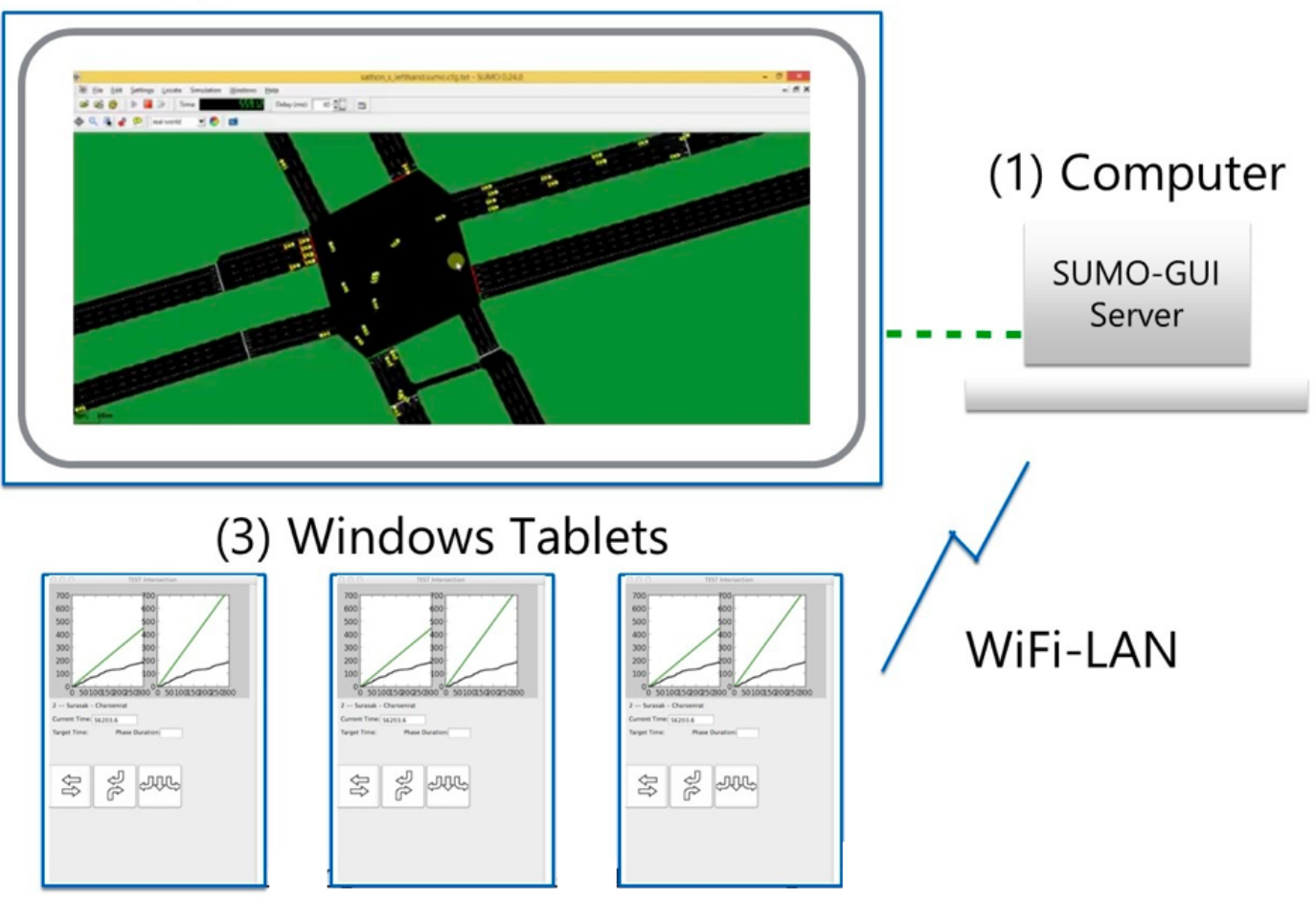

Figure 3: Chula-SSS layout of equipment settings.

actual traffic light signal control panel (shown in Figure 6) as seen by traffic police. This control panel has been recently introduced in Bangkok (Thailand) for traffic police deployment in the Chulalongkorn University's Sathorn Model project. The traffic police can here see important information, i.e., the current phase usage, the target green time and actual green time, vehicle queue length in each approaching direction at the intersection, the overall map of neighbourhood area with road segments being coloured according to travel time average updates from probed taxi vehicles, and the daily profile of vehicle flow rate of the current day vs the same day of last week.

Note also a similar plotting feature between the top part of Figure 5 and the right-hand part of Figure 6. These graphs show the real-time plot of cumulative vehicle flow rates for the flow directions getting the right-of-way at the current signal phase. The cumulative vehicle flow rates in Figures 5 and 6 are computed from the inductive loop detectors in SUMO and in the real-world installation on all approaching lanes at the stop line, respectively. Here, a flow per direction is summed up from corresponding induction loops before being plotted. In addition, the saturation flow rate collected from the past has also been depicted as a guiding linear function. This guideline is expected to help traffic police see how efficient the current green time has been used.

For instance, consider an event that the actual cumulative vehicle flow rate is much lower than the linear guideline. The first interpretation can be that the upstream road approaching that green light might have run out of vehicles or the downstream road is already jammed. In 


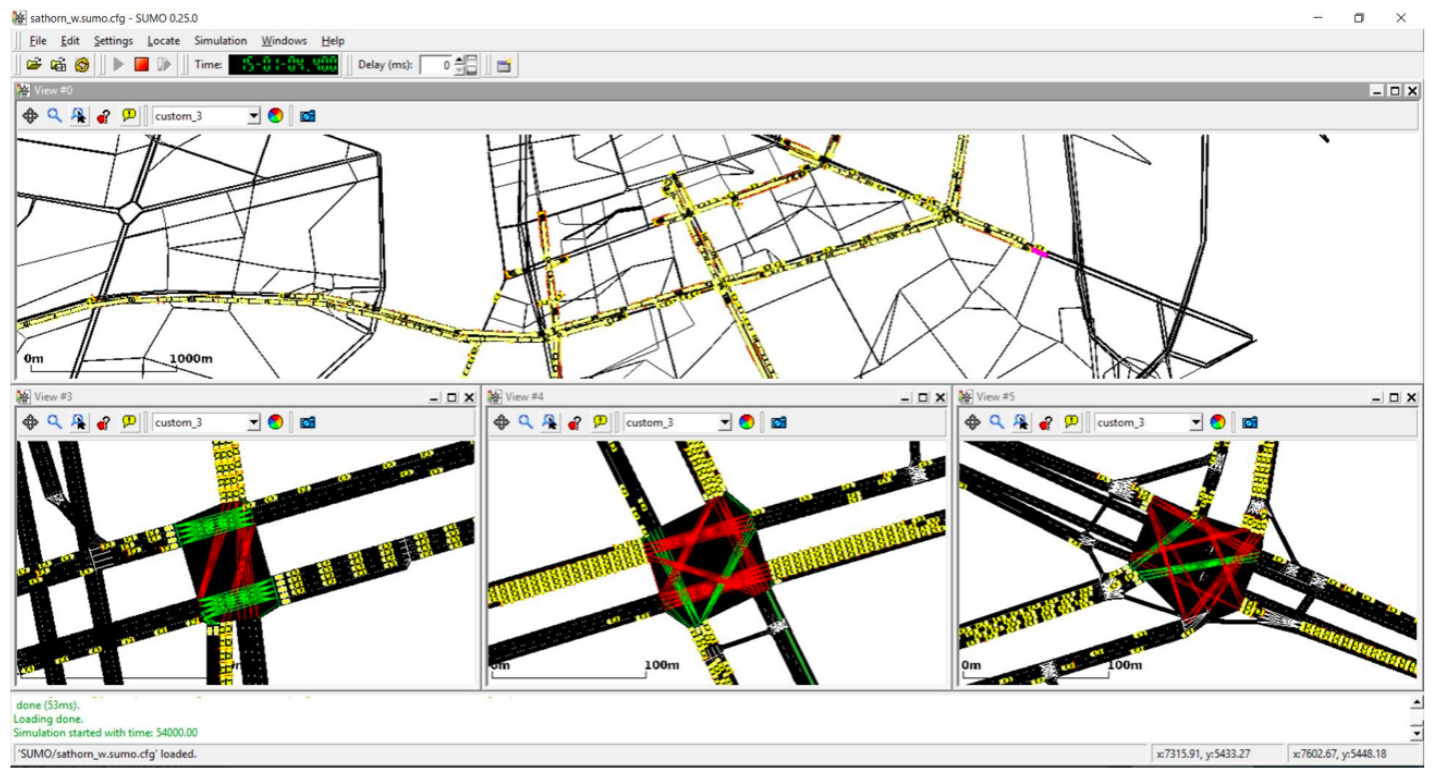

Figure 4: Chula-SSS server display of the overall Sathorn Road network (at the top) and Sathorn-Narinthorn-Wittayu Intersections (at the bottom left-middle-right).

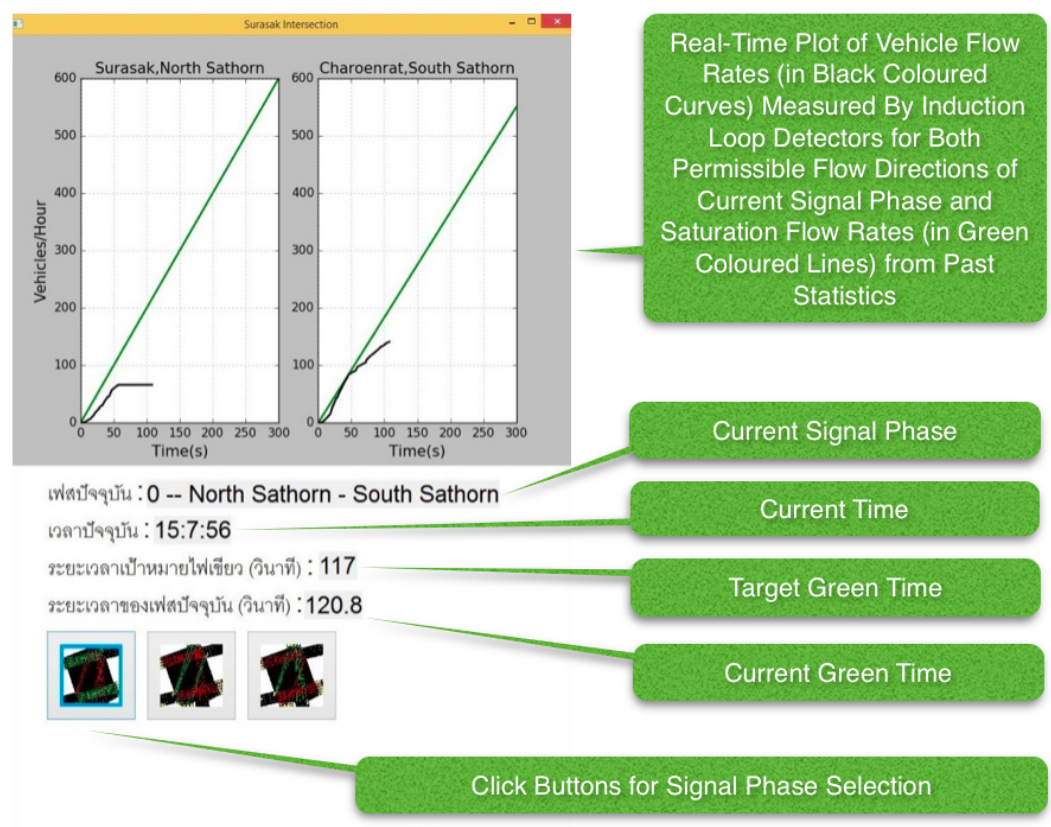

Figure 5: Chula-SSS client window at Sathorn Intersection.

any of these cases, the currently set green time should be shortened. However, given that same event but at the beginning of green time, an alternative interpretation can be a lengthened 


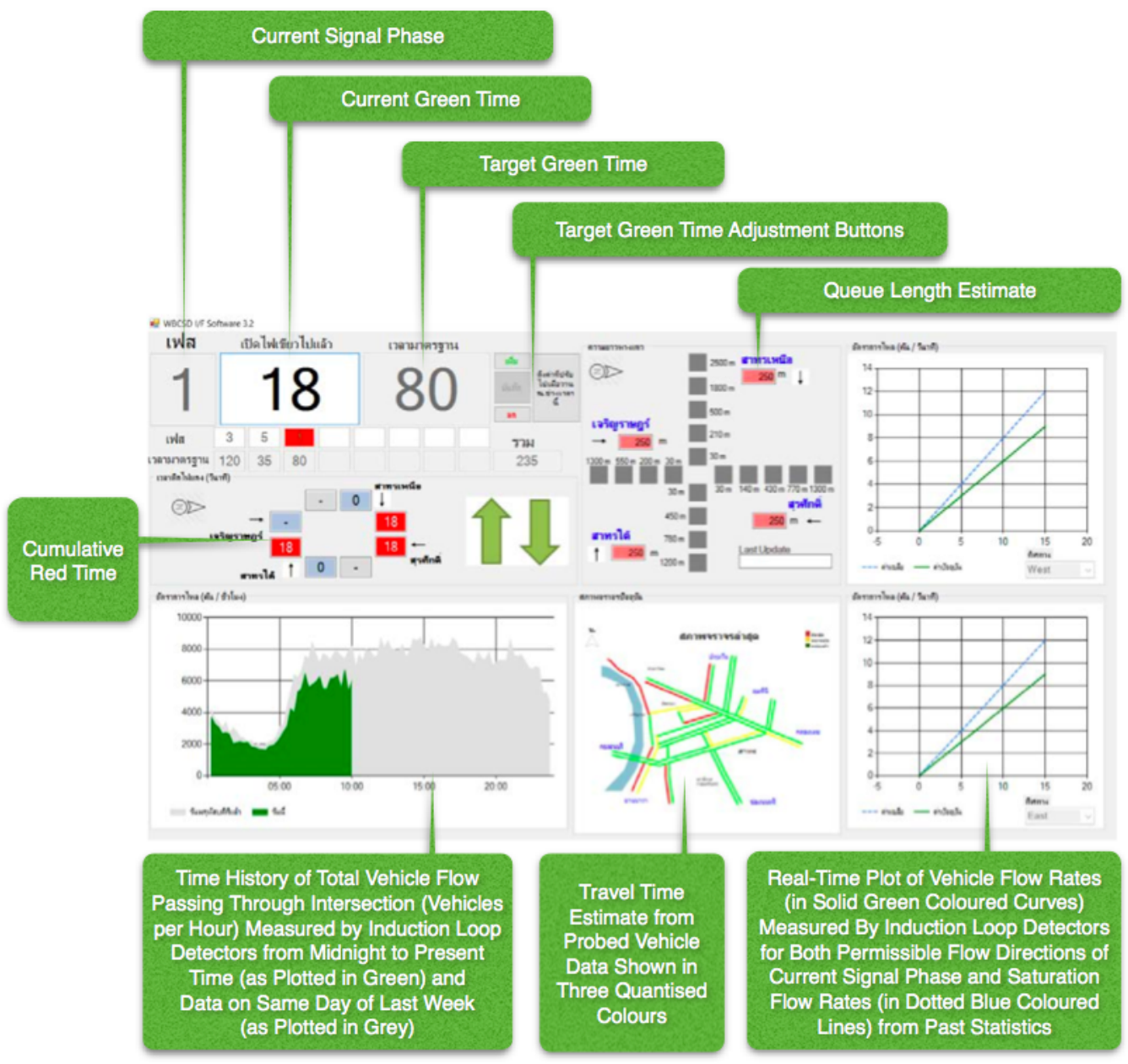

Figure 6: Local traffic control display at Sathorn Intersection.

start-up lost time. This start-up lost time can be much significant especially in a city with many motorbikes like Bangkok [9]. The signal logics must be intelligent enough to distinguish the former vs the latter interpretations. Also, based on [9], the target green time values have been optimised with Synchro software [12]. These target green time values have been displayed at Chula-SSS client windows as a suggestion for traffic police users. The analysis of start-up lost time has been thoroughly studied in [9], where SUMO is used to simulate the car mobility. At the beginning of each starting green interval, a start-up lost time is therein manually entered into SUMO by including an extra red signal phase whose interval length is set to that start-up lost time value.

The traffic light signal control panel GUI modules in Chula-SSS client can communicate via TraCI in real-time to Chula-SSS server. The purpose is to retrieve the raw traffic sensor data emulated within SUMO. Also the desired traffic light signal phase that is manually entered via GUI by the module users is sent to SUMO via TraCI. Particularly, immediately after a non-active phase button is clicked, Chula-SSS client toggles the changing phase by sending the 


\begin{tabular}{cccc}
\hline Dataset & Number of Vehicles & Simulation Time & Driving Direction \\
\hline Cologne [14] & 250 thousand & 2 hours & right-handed \\
Bologna [1] & 11 thousand & 1 hour & right-handed \\
Luxembourg [3] & 300 thousand & 24 hours & right-handed \\
Sathorn (Morning) & 55 thousand & 3 hours $(6-9$ am) & left-handed \\
Sathorn (Evening) & 55 thousand & 4 hours $(3-7 \mathrm{pm})$ & left-handed \\
\hline
\end{tabular}

Table 1: Feature comparison of Sathorn SUMO datasets and other standard datasets.

number of clicked button via a UDP socket to Chula-SSS server. Then, at Chula-SSS server, the clicked button number is converted into a string corresponding to predefined sequence of signal phase encodings and that string representing the new traffic signal state is then sent to SUMO via TraCI. By this means, a traffic light signal phase is switched immediately in SUMO simulated case when users press a new phase button. Each of the users could play a role of traffic police in charge of actuating the traffic light signal at each of the controllable intersections. All Chula-SSS programs are based on Python [8] and all GUIs have been developed with wxPython [17].

\section{Validation of Chula-SSS Framework}

\subsection{Calibrated Datasets of Sathorn Road Network}

Table 1 provides a feature comparison of Sathorn SUMO datasets and other standard datasets. The Sathorn SUMO datasets can be considered as a moderate size of traffic scenario. In the Cologne [14] and Luxembourg [3] scenarios, the network topologies of whole cities are imported into SUMO. With large amounts of traffic demand, both scenarios consume a great deal of execution time and memory resources when compared with the Sathorn SUMO datasets. A partial area of city road network in Bologna [1] is imported into SUMO, and hence a similar network size to the Sathorn SUMO datasets. There is less traffic volume in Bologna than in Sathorn scenarios. Among all these scenarios, the Sathorn scenario is the only left-handed driving case. While these other scenarios represent European city cases, our Sathorn SUMO datasets serve as a city scenario in a populous city in Southeast Asia, with distinct mobility features and over-saturated congestions that pose a challenge not only to local practitioners but also traffic engineering researcher community globally.

Chula-SSS has been implemented with two calibrated SUMO datasets for the Sathorn Road network area. This area is one of the most critical areas in Bangkok due to the immense traffic volume with daily recurring traffic bottlenecks and network deadlocks. The simulation comprises of 2375 intersection nodes, 4517 edges, 10 signalised intersections. As an example, 3 out of 10 signalised intersections have been selected for Chula-SSS client program implementation. The remaining signalised intersections are controlled by the actual traffic police operations during the day of our actual data collection.

Regarding topology construction in the Sathorn SUMO datasets, since Thailand is a lefthanded-driving country, the lefthand option in NETCONVERT is set to True. Another adjustment is in joining nodes at the intersections that use an adaptive signal control. Both Sathorn Road and Narathiwas Road have been built with canals separating between the forward and reverse movement directions. As a result, each intersection along Sathorn Road consists of two or more nodes in the initial SUMO road network representation. However, traffic light signal logic has to be defined for every node in SUMO. It is then difficult to define a proper signal logic 
encodings at those nodes corresponding to the same intersection in reality. For this reason, all the nodes at each of those intersections have been grouped into a node cluster. Further, lane connections in clusters as automatically generated in SUMO are occasionally incorrect in our case. As a corrective measure, an additional connection file has been prepared to amend lane connections in accordance with those in the real Sathorn Road network.

As usual, the dataset repository comprises of all necessary topology-definitive files. The node file contains necessary Sathorn Road network topology with the geographical coordinates properly converted to the SUMO metric system. The edge file contains the edges, defined by their upstream and downstream nodes. The link type file defines the number of lanes. The connection file defines linking lane connections from edges to adjacent downstream edges. And finally, to combine data in the node, edge, link type and connection files, the network file has been generated from NETCONVERT.

Regarding parameter calibration, the default Krauß's car-following model [5] has been used. A brute-force calibration approach has been adopted to find the optimal parameters for simulating behaviour of cars in SUMO. To reduce the degree of freedom, the maximum speed parameter is set to $12.5 \mathrm{~m} / \mathrm{s}$. This speed value has been computed from our actual data collection by considering the linearised increasing slope in the fundamental diagram. From the satellite image over the Sathorn Road, a 1000-metre road segment can contain 143 cars during the severely jammed condition. As the most popular cars in Bangkok are the Toyota Corolla Altis with the car length of $4.62 \mathrm{~m}$, subsequently, the minimum gap parameter has been equated to (1000 / 143) $-4.62 \mathrm{~m}=2.373 \mathrm{~m}$.

There remain three parameters that need be calibrated, namely, the $\sigma$ value (imperfection), acceleration, and deceleration. They have been varied from $0.0,1.0 \mathrm{~m} / \mathrm{s}^{2}$, and $1.7 \mathrm{~m} / \mathrm{s}^{2}$ to $1.0,3.0 \mathrm{~m} / \mathrm{s}^{2}$, and $3.7 \mathrm{~m} / \mathrm{s}^{2}$ with the increments of $0.1,0.2 \mathrm{~m} / \mathrm{s}^{2}$, and $0.2 \mathrm{~m} / \mathrm{s}^{2}$ respectively. So, there are $11^{3}=1,331$ combinations of parameter settings in this brute-force calibration. During the calibration, SUMO 0.25.0 has been installed in the personal computer with Intel ${ }^{\circledR}$ Core i7-4790 CPU@3.6GHz $\times 8$ and the memory of 15.6 GBytes. With Ubuntu OS 12.04.5 LTS, it has taken over 50 hours to run all 1,331 cases.

All varying combinations of these SUMO parameters have been used to find the case that minimises the summation, for all the links, of the root mean squared error (RMSE) between SUMO vs actual data collection values of link travel time as sampled every 15 -minute interval. The result is summarised in Table 2. The final parameter values found here resemble those parameter values used in [9] and [13], where the calibration purpose is to fit the SUMO simulated link travel time with that generated by another mesoscopic simulator called NETSTREAM [6] introduced by our colleagues from Toyota Central R\&D Labs in Tokyo, Japan.

For the Sathorn SUMO evening scenario (3-7 pm), the simulation period from 2.45-3 pm is used to inject flows of vehicles to store initially enough vehicles in SUMO. These backlog vehicles must match with the actual number that has been counted on the data collection day on each SUMO edge or road segment in the network. A similar treatment to match the initial vehicle backlogs has been carried out for the morning scenario. After the simulation starting time, demand on preplanned typical shortest routes has been entered. This is to match the resultant departing vehicle flow rates, sampled every 15-minute interval, from SUMO with that from the data collection day on each SUMO edge or road segment. To eliminate this initial time of running Chula-SSS, the save-and-load function can be used to initialise the traffic state right at the intended starting time like $3 \mathrm{pm}$ for the evening scenario. So, users need not wait for the transient period of injected vehicles. All the demands are given in the files of Sathorn SUMO datasets.

Visualisation tools have been developed during the calibration in Python with Bokeh [2] 


\begin{tabular}{ccc}
\hline SUMO Parameters & Sathorn (Morning) & Sathorn (Evening) \\
\hline Maximum Speed & $12.5 \mathrm{~m} / \mathrm{s}$ & $12.5 \mathrm{~m} / \mathrm{s}$ \\
Minimum Gap & $2.37 \mathrm{~m}$ & $2.37 \mathrm{~m}$ \\
$\sigma$ & 0.7 & 0.7 \\
Acceleration & $2 \mathrm{~m} / \mathrm{s}^{2}$ & $1.8 \mathrm{~m} / \mathrm{s}^{2}$ \\
Deceleration & $2.5 \mathrm{~m} / \mathrm{s}^{2}$ & $1.9 \mathrm{~m} / \mathrm{s}^{2}$ \\
\hline$R^{2}$ of link travel time sampled & & \\
every 15-minute interval from & 0.72 & 0.81 \\
SUMO vs actual data collection & & \\
\hline
\end{tabular}

Table 2: SUMO parameters found by minimising the summation, for all the links, of RMSE between SUMO vs actual data collection values of link travel time sampled every 15-minute interval.

and Plotly [7]. These two Python libraries can produce plots in HTML with rotatable graphs. Three-dimensional sensitivity analysis graphs have been plotted by setting the Z-axis to the RMSE of link travel time. The $\mathrm{X}$ and $\mathrm{Y}$ axes are set to ( $\sigma$, acceleration), ( $\sigma$, deceleration), and (deceleration, acceleration). At each $\mathrm{X}-\mathrm{Y}$ coordinate point, the value on the $\mathrm{Z}$-axis represents the average of all the RMSE values at that X-Y setting. Figure 7 depicts an example of the output results of this error sensitivity study. The black circles in this figure locate the optimal parameter settings. Due to the brute-force combinatorics of parameters being varied, this paper could account for the stochasticity of simulation result dependence on the random number generator seeds. That is, the sensitivity of resultant RMSE can be confirmed at each parametric setting point with the neighbour points with only a slight parametric value adjustment. And based on Figure 7, such error sensitivity surface contains sufficient smoothness to opt for the final combinatorial parametric selection. So, running the simulation only one time per each parametric setting case can still be reasonable. However, with a greater abundance of computing resources than that available at the time of calibration task execution in this research, a more comprehensive calibration with random seed multiplicity per each parametric setting is certainly a worthwhile future work. In addition, it is noticeable from Figure 7 that the RMSE value is more sensitive to the value of $\sigma$ than the acceleration or deceleration values. This is understandable because the calibration has been here focused on the rush hour periods in the morning and evening. Due to heavy traffic congestion, the transient behaviours affected mainly by the acceleration or deceleration parameters thus become relatively subsided. As an overall indicator for the model goodness of fit, as shown in Table 2, the correlation coefficient $R^{2}$ of link travel time as sampled every 15 -minute interval from SUMO vs actual data collection is found to be 0.72 and 0.81 for the morning and evening Sathorn SUMO datasets, respectively. This level of fitness is sufficient to validate the usage of these datasets for the intended purposes of developed Chula-SSS framework.

Finally, the calibrated Sathorn simulation has been extended to create the real scenario of parking in prohibitive areas during the evening peak hours from 3-7 pm. In that period, there would be some vehicles, like private cars, public buses, taxis or Thai Tuk Tuk cars (small traditional taxi with three wheels), stopping on South Sathorn Road near Sathorn Intersection and Narathiwas Road near Narinthorn Intersection. To include these illegal parking situation with the evening Sathorn SUMO dataset, such behaviour of stopping vehicles have been recorded by video cameras during weekdays. From the videos, the flow rate of long vehicle (bus) and normal-length vehicle (private cars, taxis and Thai Tuk Tuk cars) has been manually computed. These two vehicle types have then been added to complete the calibrated evening Sathorn 

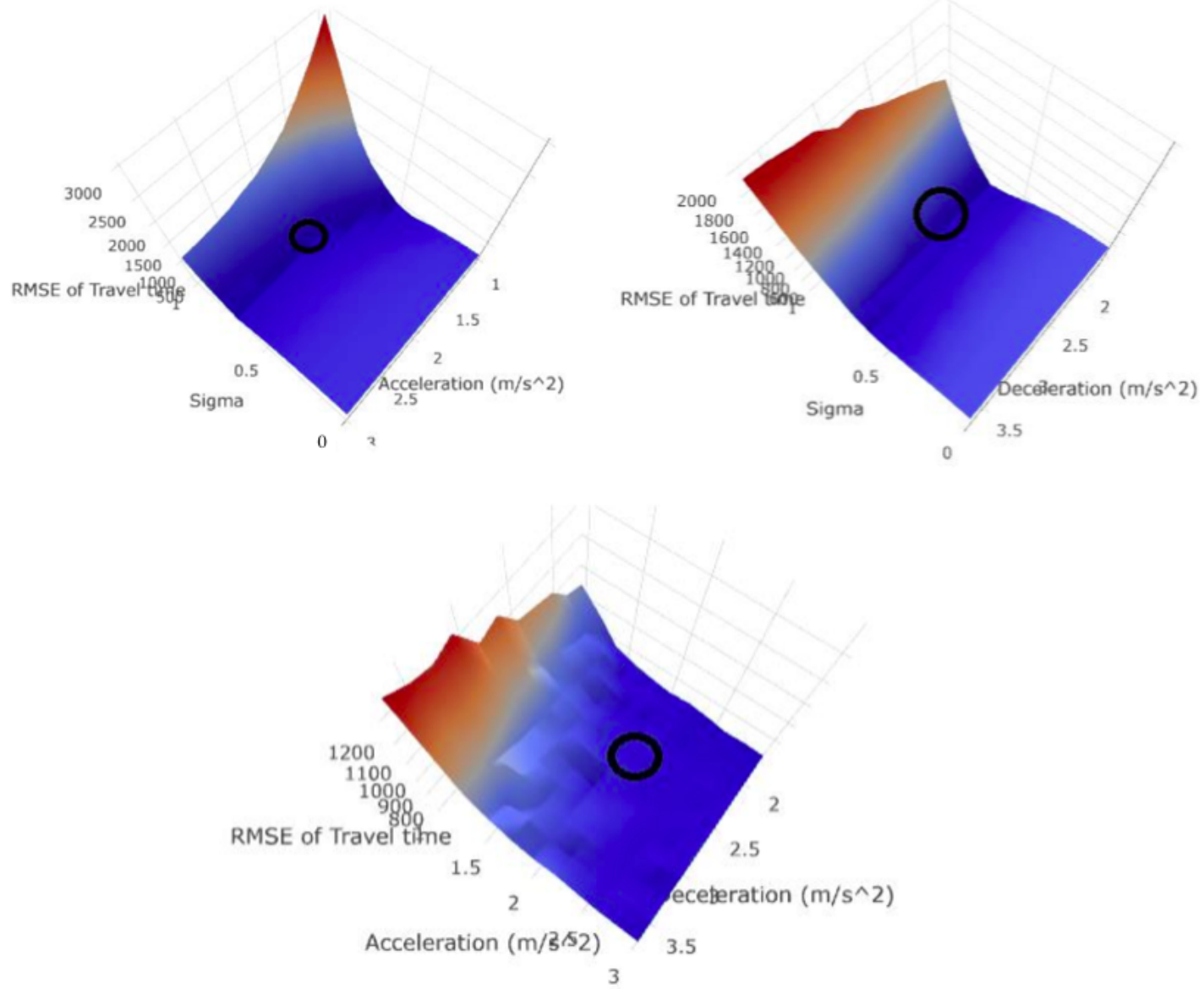

Figure 7: Sensitivity analysis for RMSE of link travel time vs pairwise-varied combinatoric values of $\sigma$, acceleration and deceleration (Sathorn morning rush hour scenario).

SUMO dataset.

\subsection{Chula-SSS in Sathorn Model Project's Social Experiment}

In the Sathorn Model project, we have developed the process to mitigate traffic congestion by involving actual commuters. The first step is the survey of citizen opinions on congestion and bottleneck mitigation ideas. We have received various ideas and those ideas have strong power to convince stakeholders. The second step is based on the on-site data collection to understand quantitatively and qualitatively the congestion situations. The third step is in brainstorming and planning of congestion mitigation measures. Here, the Chula-SSS and our calibrated Sathorn SUMO datasets have been applied to simulate various effects that would be resulted from the measures. Only the measures performing the best would then be recommended to traffic police and Bangkok Metropolitan Administration. The final step is then to carry out the implementation of the potential measures after many dialogue meetings with all stakeholders.

Within the Traffic Management and Bottleneck Mitigation Workgroup of Sathorn Model 
project, we have recommended various measures, namely, traffic signal operation adjustment, additional loop-detector sensor and video sensor installation, management of kid-dropping by parents in front of schools, relocation of bus stops, control of vehicles entering the main road from big buildings to synchronise properly with the traffic light signal of nearby intersection, law enforcement on prohibitive roadside parking area, new U-turn constructions and reversible lane operations. These measures have been tested on the real Sathorn Road network within the three-week social experiment period from Monday 6 to Sunday 26 June 2016 for evaluating their effectiveness and practicality. Once we could find effective measures, stakeholders have been recommended to continue those measures.

Chula-SSS has been demonstrated in the exhibition during the social experiment of Sathorn Model project at the Empire Tower building on Sathorn Road. The purpose of having ChulaSSS in this exhibition is to educate the general public and Sathorn Road commuters on the traffic police hardship in managing traffic congestions. Empathy and public awareness are expected so the road users understand and are willing to collaborate with the traffic police e.g. by reducing the selfishness in driving behaviours. The evening Sathorn SUMO dataset has been set as a challenging game scenario. In the game, the group of players would play the role of traffic police in controlling the traffic light signals. Since the dataset has been calibrated to mimic the real situations, the players would feel most involved and put their trials and attempts in completing the game. To ease the game completion, after injecting the vehicle backlogs into the Sathorn Road network, additional subsequent demand has been set to zero. The players must then try to clear all those vehicle backlogs as fast as they can.

Without our surprises, the group of players who have set the minimum traffic clearing time becomes the traffic police themselves. This confirms the traffic police expertise in managing traffic light signal on-site, and hence our motivation to run a knowledge management session with the traffic police team subsequently. It is apparent that, though the expert traffic police know how to practice those logics well, they cannot formalise their expertise into a standard format. Further, the current practice of traffic police is likely to be unsystematically implemented. This is understandable because different traffic police would have to take turn in staying at their signal control boxes. Without a common understanding on the signal control preferential strategy, individual traffic police operate the traffic light signals potentially differently despite of confronting by similar traffic scenarios. The problem gets worsen when traffic police need to work long hours in a stressful working environment, or upon a new traffic police workforce mobility that is carried out annually to rotate the traffic police from the current to new responsible district locations. In [13], our previous investigation has confirmed this fact by quantifying the increased average travel time at three main intersections along Sathorn Road by comparing in SUMO the case with actual manual traffic signal operations by traffic police and the case with optimal periodic signal settings by Synchro software.

To solve this problem, we need a standardisation process of traffic police's tacit know-hows in management of traffic light signals. In doing so, we have applied plausible methods ranging from Chula-SSS tools to on-site observations and mind-map sessions as well as personal interviews. Then we have created an A3-size poster summarising how the traffic light signal should be actuated upon occurrence of events e.g. queue spill backs, vehicle gap detection, maximum green time expiration, applicable traffic light signal phase sequence during each operational hour. Figure 8 depicts an output example of this process for the developed signal actuated logics during morning rush hours at Sathorn Intersection. During evening rush hours, and off-peak hours, similar diagrams have also been developed. Since the development has involved the traffic police from the start, the result has been well accepted and used in practice. 


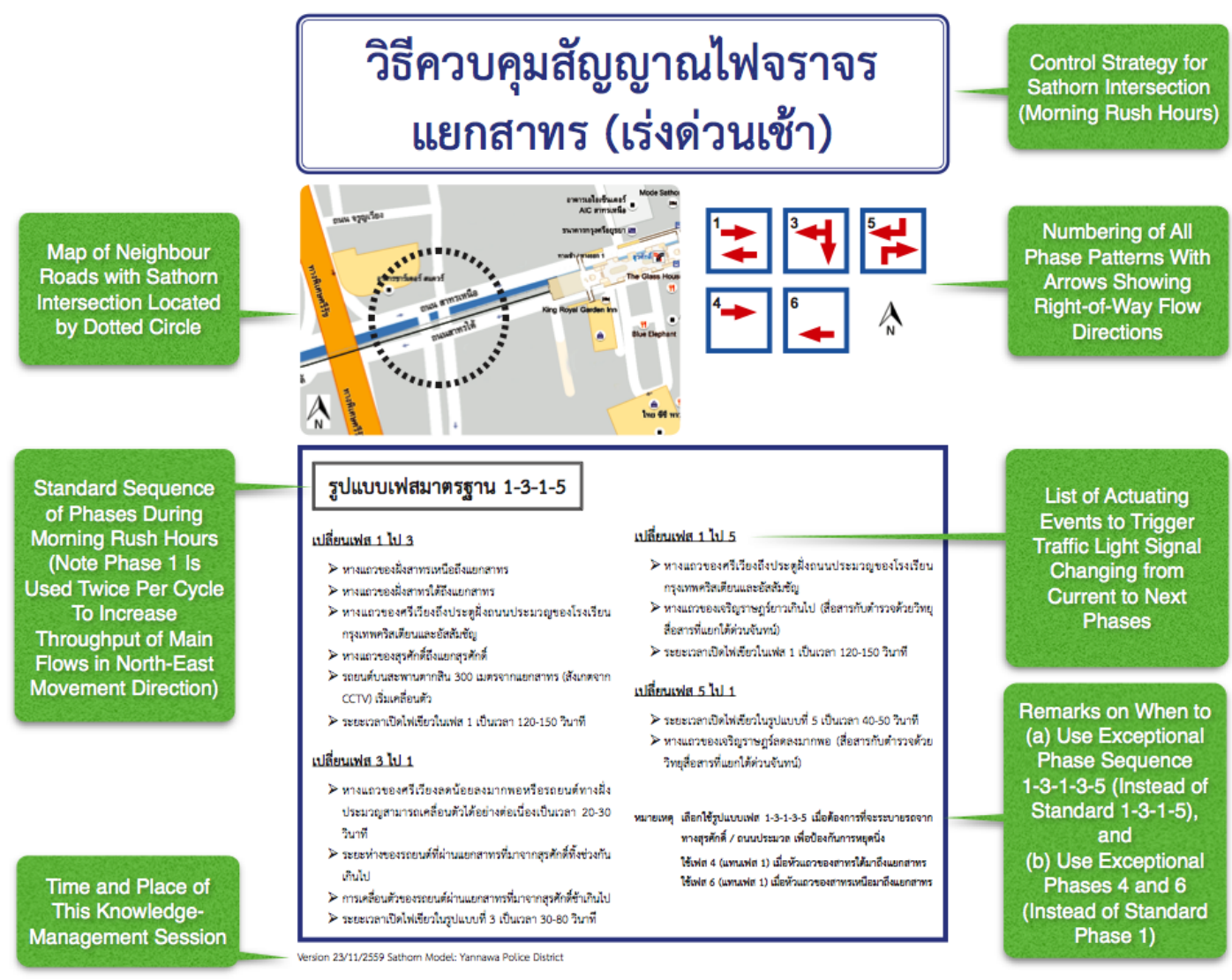

Figure 8: Knowledge-management output example of signal actuated logics standardisation during morning rush hours at Sathorn Intersection.

\section{Conclusion}

In this paper, Chula-Sathorn SUMO Simulator, or Chula-SSS, has been proposed. The aim is to incorporate the tacit traffic control expertise of human operators by trying to extract and extend the human-level intelligence in actuating logically traffic signal controls. In this regard, a new software package has been developed in SUMO. The Chula-SSS client/server programs communicate to SUMO via TraCI in real-time. The communication is to retrieve the raw traffic sensor data emulated within SUMO and to send the desired traffic light signal phase manually entered via GUI by the module users. To demonstrate this framework, Chula-SSS has been implemented with the calibrated SUMO datasets of Sathorn Road network area, one of the most over-saturated areas in Bangkok due to the immense traffic volume with daily recurring traffic bottlenecks and network deadlocks.

During the three-week social experiment period of Sathorn Model project from Monday 6 to Sunday 26 June 2016, Chula-SSS has been used successfully. It has been applied as the main educational tool for the workshop with traffic police and traffic engineers of Bangkok Metropolitan Administration. The exhibition has also been completed with Chula-SSS tools being set as a traffic signal control game. This Chula-SSS game could attract a great deal of public 
awareness especially on the traffic police hardship in having to deal with over-saturated traffic congestions. Finally, through the usage of Chula-SSS and knowledge management methodologies, a standardised strategy has been developed for actuating the traffic light signal, as an example, at the most critical Sathorn Intersection.

It is hoped that the developed framework and software package can be useful not only for our Thailand case. The case studied here is also applicable to those developing and least-developed countries where traffic signal controls rely on human operations, not yet fully automated by an area traffic controller. In those cases, the framework proposed herein is expectedly an enabling technology for the human operators to practice, learn, and evolve their traffic signal control strategies systematically. To allow a wide extension of our work, all Chula-SSS source codes and calibrated morning/evening Sathorn SUMO datasets are publicly available at https://github.com/SathornModel/Chula-SSS.

\section{Acknowledgments}

This research work has been completed during the Sustainable Mobility Project (Sathorn Model) with financial supports from Toyota Mobility Foundation. In developing and testing Chula-SSS, the authors would like to acknowledge the efforts of the following team members of Traffic Flow Management and Bottleneck Mitigation Workgroup, namely, Mr Koichi Watanabe, Dr Jittichai Rudchanakanoknad, Dr Pitipong Chanloha, Mr Apinun Intarachaiya, Mr Chadil Tuchinda, Ms Napatsorn Tantasanee, Ms Suwapatch Chantaratruxa, Ms Piyamat Pakdeumnat, Mr Suneet Kumar Singh and Mr Thanapapas Horsuwan. The authors would like to acknowledge Dr Hiroko Mori and Dr Hironobu Kitaoka for the technical supports in NETSTREAM simulator and discussions during the early version of Sathorn SUMO dataset calibration process. Lastly, the authors would like to thank the Royal Thai Police and the Traffic and Transportation Department of Bangkok Metropolitan Administration for their participations throughout the whole Sathorn Model project.

\section{References}

[1] Laura Bieker, Daniel Krajzewicz, AntonioPio Morra, Carlo Michelacci, and Fabio Cartolano. Traffic simulation for all: A real world traffic scenario from the city of bologna. In Michael Behrisch and Melanie Weber, editors, Modeling Mobility with Open Data, pages 47-60, Cham, 2015. Springer International Publishing.

[2] bokeh. https://bokeh.pydata.org. Accessed: 2018-02-11.

[3] Lara Codeca, Raphaël Frank, and Thomas Engel. Luxembourg sumo traffic (lust) scenario: 24 hours of mobility for vehicular networking research. In Vehicular Networking Conference (VNC), 2015 IEEE, pages 1-8. IEEE, 2015.

[4] Daniel Krajzewicz, Jakob Erdmann, Michael Behrisch, and Laura Bieker. Recent development and applications of SUMO - Simulation of Urban MObility. International Journal On Advances in Systems and Measurements, 5(3\&4):128-138, December 2012.

[5] Stefan Krauß. Microscopic modeling of traffic flow: Investigation of collision free vehicle dynamics. $\mathrm{PhD}$ thesis, Universitat zu Koln, 1998.

[6] Hiroko Mori, Hironobu Kitaoka, Masaya Ishida, and Yasuo Asakura. Traffic simulator netstream for evaluation of co 2 emissions. In Intelligent Transportation Systems (ITSC), 2010 13th International IEEE Conference on, pages 47-53. IEEE, 2010.

[7] plotly. https://plot.1y. Accessed: 2018-02-11.

[8] python. https://www.python.org. Accessed: 2018-02-11. 
[9] Suneet Kumar Singh, Patrachart Komolkiti, and Chaodit Aswakul. Impact analysis of start-up lost time at major intersections on sathorn road using a synchro optimization and a microscopic sumo traffic simulation. IEEE Access, 2017.

[10] TomTom NV Dutch Company, Amsterdam, The Netherlands. Tomtom traffic index. https: //www.tomtom.com/en_gb/trafficindex/city/bangkok, March 2016. Accessed: 2018-02-11.

[11] Traffic and Transportation Department. Traffic statistics 2015. Technical report, Bangkok Metropolitan Administration, 2016.

[12] Trafficware, Sugar Land, TX, USA. Synchro studio 9.

[13] Kijawattanee Udomsilp, Teeratat Arayakarnkul, Sorawee Watarakitpaisarn, Patrachart Komolkiti, Jittichai Rudjanakanoknad, and Chaodit Aswakul. Traffic data analysis on sathorn road with synchro optimization and traffic simulation. Engineering Journal (Eng. J.), 21(6):57-67, 2017.

[14] Sandesh Uppoor and Marco Fiore. Large-scale urban vehicular mobility for networking research. In Vehicular Networking Conference (VNC), 2011 IEEE, pages 62-69. IEEE, 2011.

[15] Axel Wegener, Michał Piórkowski, Maxim Raya, Horst Hellbrück, Stefan Fischer, and Jean-Pierre Hubaux. Traci: an interface for coupling road traffic and network simulators. In Proceedings of the 11th communications and networking simulation symposium, pages 155-163. ACM, 2008.

[16] World Business Council Sustainable Development, Geneva, Switzerland. Integrated sustainable mobility in cities-a practical guide, sustainable mobility project 2.0. http://www.wbcsd.org/Projects/SiMPlify/Resources/SMP2. 0-Final-Report-Integrated-Sustainable-Mobility-in-Cities-a-practical-guide, April 2016. Accessed: 2018-02-11.

[17] wxpython. https://www.wxpython.org. Accessed: 2018-02-11. 